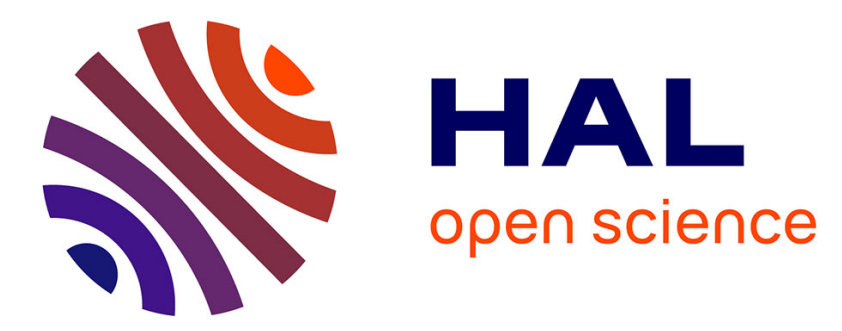

\title{
Classification of 3T1R Parallel Manipulators Based on Their Wrench Graph
}

\author{
Semaan Amine, Ossama Mokhiamar, Stéphane Caro
}

\section{To cite this version:}

Semaan Amine, Ossama Mokhiamar, Stéphane Caro. Classification of 3T1R Parallel Manipulators Based on Their Wrench Graph. Journal of Mechanisms and Robotics, 2017, 9 (1), 10.1115/1.4035188 . hal-01721206

\section{HAL Id: hal-01721206 https://hal.science/hal-01721206}

Submitted on 1 Mar 2018

HAL is a multi-disciplinary open access archive for the deposit and dissemination of scientific research documents, whether they are published or not. The documents may come from teaching and research institutions in France or abroad, or from public or private research centers.
L'archive ouverte pluridisciplinaire HAL, est destinée au dépôt et à la diffusion de documents scientifiques de niveau recherche, publiés ou non, émanant des établissements d'enseignement et de recherche français ou étrangers, des laboratoires publics ou privés. 


\title{
Classification of 3T1R Parallel Manipulators Based on Their Wrench Graph
}

\author{
Semaan Amine* \\ Department of Mechanical Engineering, \\ Beirut Arab University, \\ P.O. Box: 115020, \\ Riad El Solh' 11072809, \\ Beirut, Lebanon \\ Email: s.amin@bau.edu.lb \\ Stéphane Caro \\ Institut de Recherche en Communications \\ et Cybernétique de Nantes, \\ UMR CNRS no. 6597 , \\ 1 rue de la Noë, 44321 , \\ Nantes, France \\ Email: stephane.caro@irccyn.ec-nantes.fr
}

Ossama Mokhiamar

Department of Mechanical Engineering,

Beirut Arab University,

P.O. Box: 115020,

Riad El Solh 11072809,

Beirut, Lebanon

Department of Mechanical Engineering,

Alexandria University,

El-Chatby, Alexandria 21544,

Alexandria, Egypt

Email: ossama.mokhiamar@bau.edu.lb

\begin{abstract}
This paper presents a classification of $3 T 1 R$ parallel manipulators based on the wrench graph. By using the theory of reciprocal screws, the properties of the three-dimensional projective space, the wrench graph and the superbracket decomposition of Grassmann-Cayley algebra; six typical wrench graphs for 3T1R parallel manipulators are obtained along with their singularity conditions. Furthermore, the paper shows a way in which each of the obtained typical wrench graphs can be used in order to synthesize new $3 T 1 R$ parallel manipulator architectures with known singularity conditions and with an understanding of their geometrical properties and assembly conditions.
\end{abstract}

\section{Introduction}

Parallel Manipulators (PMs) whose moving platform performs 3T1R motions, namely, three independent translations and one rotation about an axis of fixed direction [1], are called Schoenflies Motion Generators (SMGs). This set of displacements was first studied by the German mathematician-mineralogist Arthur Moritz Schoenflies (1853-1928). Over the past few decades, the creation of various designs of 3T1R PMs was broaden, especially after the huge success of the Quattro [2]. Gogu discovered an isotropic architecture (its Jacobian matrix is diagonal and constant), named the Isoglide4 [3,4], which is composed of four legs with prismatic actuators. Another topology within the same family was introduced by Gosselin [5], named the Quadrupteron.

The symmetrical design was proposed by Angeles [1], namely the McGill SMG, with two identical legs which, in turn, decreases the number of joints. There exist two architecture varieties of $\mathrm{H} 4$ family, either with revolute or with prismatic actuators [6-8]. The $\mathrm{H} 4$ robot designed by Pierrot $e t$ al. [6,7], is a fully-parallel mechanism with no passive kinematic chain between the base and the nacelle. This idea brought out the mechanism with four legs. Each revolute joint in the leg is actuated. The $\mathrm{H} 4$ robot with prismatic actuators mounted to the base, was presented by Wu et al. [8]. Another mechanism constructed by three identical legs was proposed by Briot and Bonev $[9,10]$, called Pantopteron- 4 , having a pantograph

* Address all correspondence to this author. 
linkage in each leg. Since it only employs three legs, the Pantopteron-4 gains great advantage in terms of workspace size and acceleration capacities. Xie and Liu [11] designed the X4 robot, a high-speed 3T1R PM with high rotational capability. Wu et al. [12] addressed the multi-objective design optimization of a 3T1R PM with consideration of the kinematic and dynamic performance. Carricato [13] took advantage of the theory of reciprocal screws in order to design singularity-free SMGs. Actuation redundancy [14] is interesting for singularity and obstacle avoidance, for improvement in force control and for kinematic calibration. Recently, several parallel mechanisms with actuation redundancy were proposed $[15,16]$.

The type synthesis approach based upon screw theory is widely used for generating lower-mobility PMs as shown by Huang and Li [17, 18] and by Kong and Gosselin [19]. Based upon the reciprocity condition, Ling and Huang [20] and then Joshi and Tsai [21] developed a procedure to express the overall Jacobian matrix $\mathbf{J}$ of limited degree of freedom (dof) PMs, comprising both constraint and actuation wrenches. The rows of $\mathbf{J}$ are composed of $n$ linearly independent actuation wrenches plus (6- $n$ ) linearly independent constraint wrenches. These wrenches correspond to six Plücker lines, composing $\mathbf{J}$. The determinant of $\mathbf{J}$ is equal to the superjoin of 6 Plücker lines, named superbracket of Grassmann-Cayley algebra. It allows a translation of synthetic geometric conditions into invariant (coordinate-free) algebraic expression.

The superbracket decomposition was employed by Ben-Horin and Shoham [22,23] to analyse the singularity of 6dof PMs, for instance Gough-Stewart Platforms. However, this procedure does not consider the plane at infinity, which obviously cannot represent a line at infinity. Indeed, a line at infinity correlates to a pure moment emerged from limited dof PMs. Points at infinity were used initially in a superbracket by Kanaan et al [24]. This improvement enhanced the application of Grassmann-Cayley algebra for limited dof PMs. The method was later expanded to represent the wrenches in a projective space, named wrench graph by Amine et al [25-28]. The wrench graph depicts all geometric properties between the constraint and actuation wrenches of manipulators and highlights points at infinity in a superbracket.

This paper presents an extensive investigation of 3T1R PMs and introduces a procedure that can be followed to build and validate new 3T1R parallel robot architectures by using prismatic and/or revolute joints. First, the list of limbs for 3T1R PMs obtained in [19] is reformulated by considering the rotational dof of the moving platform to be about an axis directed along $\mathbf{z}$. The constraint wrench systems for each limb are then investigated and the influence of the limb dof and topology on the constraint singularity of the 3T1R PM is explained. Furthermore, some limb arrangements that can be used to synthesize 3T1R PMs free of constraint singularities are highlighted. Second, the actuation wrenches generated by numerous types of limbs, with one actuated joint, are listed. Moreover, the validity rules for the actuation wrench system of a proposed 3T1R PM are introduced. Those rules can be used to validate any assembly of limbs, namely, to check whether a given assembly of limbs can result in a PM whose moving platform would be able to generate a 3T1R PM in a general non-singular configuration. Third, a classification of 3T1R PMs is presented and six typical wrench graphs are introduced. This classification is based on the actuation wrenches applied by the limbs and on the resulting wrench graph. The concept of wrench graph consequently completes the type synthesis process (based on screw theory) at the conceptual design stage. This idea will be applied in this paper to illustrate all the constraint and actuation wrenches. It allows to construct new architectures of 3T1R PMs based on the classification of wrench graph and the singularity conditions. Finally, the results are verified on some existing 3T1R PMs and some other ones introduced in this paper based on the proposed procedure. Accordingly, the paper shows how each of the six typical wrench graphs can be used in order to build a valid 3T1R PM architecture and allows the consideration of parallel singularities at the conceptual design stage. In addition, some 3T1R PMs with reduced singularities are introduced.

\section{Type Synthesis of 3T1R PMs}

The moving platform of a 3T1R PM provides three independent translational dof and one rotational dof about an axis of fixed direction. In this paper, it is considered that the rotational dof is about an axis directed along $\mathbf{z}$.

\subsection{Constraint Wrench System}

In a general configuration, the constraint wrench system, $\mathrm{W}_{c}$, of a 3T1R PM must be reciprocal to the 3T1R motion. Therefore, it is a $2-\$_{\infty}$-system containing infinite-pitch wrenches (pure moments) whose directions are orthogonal to $\mathbf{z}$. Accordingly, $\mathrm{W}_{c}$ can be written as:

$$
\mathrm{W}_{c}=\operatorname{span}\left(\hat{M}_{c 1}, \hat{M}_{c 2}\right)
$$

where $\hat{M}_{c 1}=\left(\mathbf{0}, \mathbf{z} \times \mathbf{m}_{1}\right), \hat{M}_{c 2}=\left(\mathbf{0}, \mathbf{z} \times \mathbf{m}_{2}\right)$ and $\mathbf{z}, \mathbf{m}_{1}$ and $\mathbf{m}_{2}$ are three mutually independent unit vectors.

The constraint moment $\hat{M}_{c 1}$ corresponds to a line at infinity passing through points $\underline{j}=(\mathbf{z}, 0)$ and $\underline{i}=\left(\mathbf{m}_{1}, 0\right)$. Therefore, $\hat{M}_{c 1}=\underline{j} \underline{i}$. Likewise, $\hat{M}_{c 2}=\underline{j} \underline{\mathrm{k}}$ where $\underline{\mathrm{k}}=\left(\mathbf{m}_{2}, 0\right)$. As a result, the constraint wrench $\operatorname{system} \mathrm{W}_{c}=\operatorname{span}\left(\hat{M}_{c 1}, \hat{M}_{c 2}\right)$ corresponds (in a general configuration) to a flat pencil containing all lines at infinity through point $\underline{j}=(\mathbf{z}, 0)$, $\mathbf{z}$ being parallel to the axis of the allowed rotation of the PM's moving platform. 


\subsubsection{Constraint Singularities}

The constraint singularities of a 3T1R PM correspond to configurations in which the constraint wrench system fails to be a 2-system corresponding to a flat pencil of lines at infinity through point $j$. In such configurations, the PM can switch to another motion mode whose motion pattern is no longer a 3T1R motion. The change in the motion type is a phenomenon that is present in reconfigurable PMs [29] and metamorphic PMs [30,31].

However, if the constraint wrench system of a 3T1R PM corresponds to the 2-system in any configuration, then the PM will be free of constraint singularities. Therefore, it is possible to avoid such configurations with an appropriate choice of the PM's architecture, as shown thereafter in this paper.

\subsection{Actuation Wrench System}

In a general configuration, by locking all the actuated joints of a PM its moving platform must be fully constrained, i.e., its actuation wrench system must span in addition to the constraint wrench system a 6-system. Therefore, the actuation wrench system $\mathrm{W}_{a}$ of a 3T1R PM must be (in a general configuration) a 4-system and the linear combination of $\mathrm{W}_{c}$ and $\mathrm{W}_{a}$ must lead to a 6-system, otherwise the robot is permanently singular.

\subsubsection{Actuation Singularities}

The actuation singularities occur when the actuation wrench system of the PM does not satisfy any longer the previous condition while the PM is not in a constraint singularity. Therefore, a 3T1R PM exhibits an actuation singularity whenever:

- $\mathrm{W}_{a}$ fails to be a 4-system;

- the linear combination of $\mathrm{W}_{a}$ and $\mathrm{W}_{c}$ fails to be a 6-system while $\mathrm{W}_{c}$ is a 2-system.

\subsection{Comparison of Limbs for 3T1R PMs}

A list of 4- and 5- dof limbs for 3T1R PMs with R- and/or P-joints was obtained in [19]. Since the rotational dof of the 3T1R PMs under study is considered to be about an axis directed along $\mathbf{z}$, the following conditions ${ }^{1}$ should necessarily be satisfied:

- In a given limb, the axes of the R-joints must lie in parallel planes;

- If the axes of all the R-joints in a limb are parallel, then they must necessarily be directed along $\mathbf{z}$;

In order to highlight the axis direction of a revolute joint, let:

1. R denote a revolute joint of axis parallel to $\mathbf{z}$;

2. Ŕ can be:

(a) a revolute joint, $\overline{\mathrm{R}}$, of axis parallel to the horizontal plane $(\mathbf{x} O \mathbf{y})$;

(b) a revolute joint $\tilde{\mathrm{R}}$ whose axis is neither parallel to $\mathbf{z}$ nor to the horizontal plane $(\mathbf{x} O \mathbf{y})$.

3. In the $i$ th limb, let $\mathbf{m}_{i}$ denote the unit vector along the direction of the revolute joint axes that are not parallel to $\mathbf{z}$;

Therefore, all $\overline{\mathrm{R}}$-joints (all $\tilde{\mathrm{R}}$-joints, respectively) in a given limb have parallel axes. Now let us reformulate the list of limbs for 3T1R PMs obtained in [19] under the above assumptions. Accordingly, Table 1 is obtained. Here, this list is examined by a comparative analysis of the limbs in terms of both constraint and actuation wrench systems.

It is noteworthy that because of permutations, two revolute joints that are required to be parallel may become not adjacent. In particular, a revolute joint $\mathrm{R}_{j}$ on the moving platform may be required to be parallel to a revolute joint $\mathrm{R}_{i}$ on the fixed base. Here, prismatic joints are not taken into consideration because they do alter the orientation of the links that they are connected to. If $\mathrm{R}_{i}$ and $\mathrm{R}_{j}$ are parallel to $\mathbf{z}$, it will not cause any problem, since $\mathrm{R}_{j}$ will remain parallel to $\mathbf{z}$ for any Schoenflies motion of the moving platform. However, if $\mathrm{R}_{j}$ is not parallel to $\mathbf{z}$, the parallelism with $\mathrm{R}_{i}$ will not be preserved for finite displacements. Accordingly, permutations leading to this situation should be excluded.

\subsection{Limb Constraint Wrench System}

The constraint wrench system of a given limb only depends on the joint types and the joint axes. However, the arrangement of the joints within the limb may affect the constraint singularities of the obtained 3T1R PM.

\footnotetext{
${ }^{1}$ Note that these conditions rely on the assumption that the limb does not contain idle joints, i.e., joints whose kinematic elements do not undergo relative motions for finite displacements of the mechanism
} 
Table 1. Limbs for 3T1R PMs.

\begin{tabular}{|c|c|c|}
\hline$d o f$ & Class & Type \\
\hline \multirow[t]{3}{*}{4} & 3R-1P & Permutation of PR̀R̀ \\
\hline & $2 \mathrm{R}-2 \mathrm{P}$ & Permutation of PPRR \\
\hline & $1 \mathrm{R}-3 \mathrm{P}$ & Permutation of PPPR \\
\hline \multirow[t]{8}{*}{5} & $5 \mathrm{R}$ & Permutation of R̀R̀ŔŔ \\
\hline & & Permutation of RेRेŔŔR \\
\hline & 4R-1P & Permutation of PRेR̀Ŕ \\
\hline & & Permutation of PR̀RŔŔ \\
\hline & & Permutation of PRŔŔŔ \\
\hline & $3 \mathrm{R}-2 \mathrm{P}$ & Permutation of PPRेR̀R \\
\hline & & Permutation of PPRŔŔ \\
\hline & $2 \mathrm{R}-3 \mathrm{P}$ & Permutation of PPPRंR \\
\hline
\end{tabular}

\subsubsection{Four-dof Limbs}

Any 4-dof limb of Table 1 applies a $2-\$_{\infty}$-system of constraint wrenches reciprocal to the $\mathrm{R}$-joints of the limb that are directed along $\mathbf{z}$. Therefore, such a limb applies, in any configuration, an infinite number of constraint moments that correspond to lines at infinity passing through point $\underline{j}=(\mathbf{z}, 0)$. It can be concluded that a 3T1R PM having at least one 4-dof limb of Table 1 is over-constrained and free of constraint singularity.

It should be noted that for the 4-dof limbs given in Table 1 the directions of the $\mathrm{P}$-joints are defined in such a way that the limb generates a 3T1R motion. For example, let us consider the PRेR limb type. The three $\dot{R}$-joints generate, in a general configuration, one rotation about an axis directed along $\mathbf{z}$ and two translations along directions orthogonal to $\mathbf{z}$. Therefore, the direction of the $\mathrm{P}$-joint needs a nonzero component along $\mathbf{z}$, otherwise the limb cannot provide the required 3-dof translational motions.

\subsubsection{Five-dof Limbs}

A 5-dof limb used in the topology of a 3T1R PMs must apply one constraint moment that corresponds to a line at infinity passing through point $j=(\mathbf{z}, 0)$. Accordingly, the 3T1R PM must be assembled such that any 5-dof limb of the PM provides a 3T2R motion in the starting configuration. The 5-dof limbs given in Table 1 can be classified into two types:

Type 1 There exist within the limb two revolute joints of parallel axes that are neither successive nor separated by a $P$-joint. Such a limb is not generally a 3T2R limb. The 3T1R PM containing limbs of this type is obtained by imposing some geometric constraints on the assembly.

Type 2 Two revolute joints of parallel axes within the limb are either successive or separated by a $P$-joint. Moreover, the revolute joints that are not parallel to $\mathbf{z}$ are assembled on the platform. Such a limb is generally a 3T2R limb.

In order to highlight the difference between the two types of limbs let us consider two 3T1R PMs with identical limb

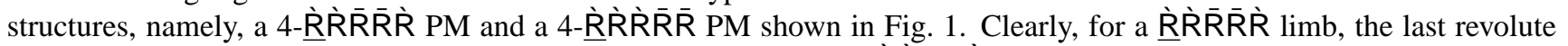
joint axis is not necessarily parallel to the first two. Therefore, the 4- $\underline{R} \dot{R} \bar{R} \bar{R} \dot{R} 3 T 1 R$ PM is obtained by assembling the PM such that:

- the condition of parallelism between the last revolute joint axis and the first two is satisfied for all limbs;

- vectors $\mathbf{m}_{i}, i=1, \ldots, 4$, are not all parallel.

In that case, the limbs apply altogether four constraint wrenches that correspond to four lines at infinity passing through point $j$ and span a 2-system of constraint wrenches. However, if the four vectors $\mathbf{m}_{i}$ become parallel, the PM will exhibit a constraint singularity and can switch to another motion mode in which the motion of the moving platform will no longer be a 3T1R motion. In such a configuration, the last revolute joint axis in each limb of the 4- $\underline{R} \dot{R} \bar{R} \bar{R} R$ PM will no longer be parallel to $\mathbf{z}$ and the moving platform will no longer be parallel to the horizontal plane $(\mathbf{x} O \mathbf{y})$.

Now let us consider the 4- $\underline{R} \dot{R} \hat{R} \bar{R} \bar{R}$ PM. Clearly, its limbs do not need geometric assembly conditions in order to provide a 3T2R motion for the limb and a 3T1R motion for the moving platform. This PM is free of constraint singularities and the moving platform is always parallel to plane $(\mathbf{x} O \mathbf{y})$. Moreover, it can be concluded that if a 3T1R PM contains at least two 


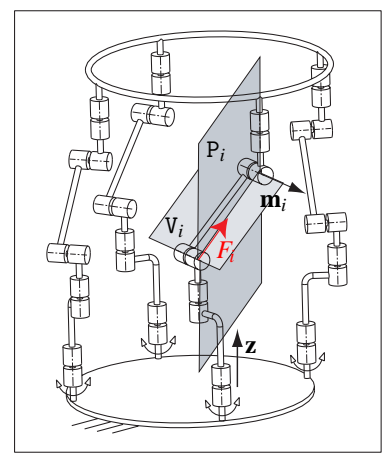

(a) $4-\underline{\hat{R}} \dot{R} \bar{R} \bar{R} \grave{R}$

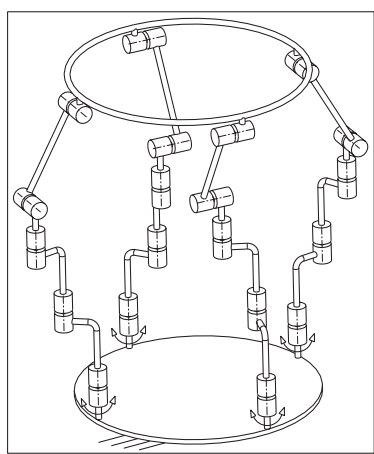

(b) $4-\underline{R} \dot{R} \dot{R} \bar{R} \bar{R}$

Fig. 1. Two 3T1R PMs [19].

limbs of type 2, its moving platform will always be parallel to plane (xOy). Therefore, the PM will be free of constraint singularities. However, if all the limbs of the 3T1R PM are of type 1, then the PM may have some constraint singularities.

\subsection{Limb Actuation Wrench System}

For a given type of limbs given in Table 1, the actuation wrenches do not depend on the arrangement of the joints within the limb but on the actuated joints. The constraint wrench system $\mathrm{W}^{c}$ of a limb is reciprocal to all the twists associated with the limb's kinematic joints. If the actuated joint of the limb is locked, then the wrench system reciprocal to the other joints of the limb will be given by $\mathrm{U}^{c}$ such that: $\operatorname{dim}\left(\mathrm{U}^{c}\right)=\operatorname{dim}\left(\mathrm{W}^{c}\right)+1$ and $\mathrm{U}^{c}$ contains $\mathrm{W}^{c}$ plus a set of some additional wrenches. Then, the corresponding actuation wrench can be selected as one of these additional wrenches.

Table 2 characterizes the locus of the actuation wrench for each type of limbs obtained in Table 1 and for each type of actuated joints. For convenience, it is considered that one joint per limb is actuated.

\subsection{Validity Rules for the Actuation Wrench System}

In what follows, let us assume that the limbs apply a 2-system of constraint wrenches spanned by $\hat{M}_{c 1}$ and $\hat{M}_{c 2}$ and satisfying Eqn. (1). In this section, we develop some rules to examine the validity of the actuation wrench system of a 3T1R PM. For instance, we consider that an actuation wrench applied by a given limb of a PM is a zero- or an infinite-pitch wrench namely, a pure force or a pure moment. Let $\hat{F}_{1}, \ldots, \hat{F}_{4}$ denote actuation forces and $\hat{M}_{a}, \hat{M}_{a 1}$ and $\hat{M}_{a 2}$ denote actuation moments. In a general configuration of a 3T1R PM, one can state the following rules.

R1 ${ }^{2}$ A basis of the actuation wrench system $\mathrm{W}^{a}$ can contain at most one actuation moment.

Proof Let us consider that a basis of $\mathrm{W}^{a}$ contains two actuation moments $\hat{M}_{a 1}$ and $\hat{M}_{a 2}$. In that case, $\mathrm{W}^{a}+\mathrm{W}^{c}=$ $\operatorname{span}\left(\hat{F}_{1}, \hat{F}_{2}, \hat{M}_{a 1}, \hat{M}_{a 2}, \hat{M}_{c 1}, \hat{M}_{c 2}\right)$. Since $\operatorname{dim}\left(\operatorname{span}\left(\hat{M}_{a 1}, \hat{M}_{a 2}, \hat{M}_{c 1}, \hat{M}_{c 2}\right)\right) \leq 3$, the dimension of $\mathrm{W}^{a}+\mathrm{W}^{c}$ will be lower than or equal to 5 in any robot configuration and therefore, the proposed PM will be permanently singular.

R2 If a basis of $\mathrm{W}^{a}$ contains one actuation moment $\hat{M}_{a}$, then the line at infinity corresponding to $\hat{M}_{a}$ should not pass through point $\underline{j}=(\mathbf{z}, 0)$.

Proof Let us assume that a basis of $\mathrm{W}^{a}$ contains one actuation moment $\hat{M}_{a}$ that corresponds to a line at infinity passing through point $\underline{j}$, in a general configuration. In that case, the actuation moment $\hat{M}_{a}$ belongs to the constraint wrench system in a general configuration. Clearly in that case: $\operatorname{dim}\left(W^{a}+W^{c}\right) \leq 5$.

R3 If the basis of $\mathrm{W}^{a}$ contains one actuation moment $\hat{M}_{a}$ and three actuation forces $\hat{F}_{1}, \ldots, \hat{F}_{3}$, then none of the three actuation forces can be parallel to another.

Proof Indeed, if $\hat{F}_{1}$ and $\hat{F}_{2}$ are parallel, then $\operatorname{span}\left(\hat{F}_{1}, \hat{F}_{2}\right)$ is equivalent to $\operatorname{span}\left(\hat{F}_{1}, \hat{M}_{12}\right), \hat{M}_{12}$ being the line at infinity of the finite plane containing $\hat{F}_{1}$ and $\hat{F}_{2}$. Accordingly, one can find a basis of $\mathrm{W}^{a}$ containing two actuation moments, and therefore, rule $\mathbf{R} \mathbf{1}$ is not satisfied in that case.

$\mathbf{R} 4$ If a basis of $\mathrm{W}^{a}$ contains one actuation moment $\hat{M}_{a}$ and three actuation forces $\hat{F}_{1}, \ldots, \hat{F}_{3}$, then the three actuation forces cannot be coplanar.

Proof For instance, if $\hat{F}_{1}, \hat{F}_{2}$ and $\hat{F}_{3}$ are coplanar and do not pass through the same finite point, then $\operatorname{span}\left(\hat{F}_{1}, \hat{F}_{2}, \hat{F}_{3}\right)$ will be equivalent to $\operatorname{span}\left(\hat{F}_{1}, \hat{F}_{2}, \hat{M}_{a 1}\right), \hat{M}_{a 1}$ being the line at infinity of the finite plane containing $\hat{F}_{i}(i=1, \ldots, 3)$. Accordingly, rule $\mathbf{R} 1$ is not satisfied in that case.

On the other hand, if $\hat{F}_{1}, \hat{F}_{2}$ and $\hat{F}_{3}$ are coplanar and do pass through the same finite point, then $\operatorname{span}\left(\hat{F}_{1}, \hat{F}_{2}, \hat{F}_{3}\right)$ will fail to be a 3-system.

\footnotetext{
${ }^{2} \mathbf{R 1}$ stands for rule 1
} 
Table 2. Actuation Wrench of Limbs for 3T1R PMs With one Actuated Joint.

\begin{tabular}{|c|c|c|c|}
\hline$d o f$ & Type & $\begin{array}{l}\text { Actuated } \\
\text { joint }\end{array}$ & Actuated wrench \\
\hline \multirow[t]{6}{*}{4} & \multirow{2}{*}{$\begin{array}{l}\text { Permutation of PRेR̀ } \\
\text { (the P-joint being } \\
\text { along } \mathbf{z} \text { ) }\end{array}$} & $\underline{P}$ & A force directed along $\mathbf{z}$ \\
\hline & & $\underline{\mathrm{R}}$ & $\begin{array}{l}\text { A force orthogonal to } \mathbf{z} \text { and lying in the plane containing the axes of two unactuated } \\
\text { R-joints }\end{array}$ \\
\hline & \multirow[t]{2}{*}{ Permutation of PPR̀R } & $\underline{P}$ & $\begin{array}{l}\text { A force not orthogonal to the direction of the actuated } \underline{P} \text {-joint, orthogonal to the } \\
\text { direction of the actuated } \underline{P} \text {-joint and lying in the plane containing the axes of two } \\
\text { unactuated } R_{\text {-joints }}\end{array}$ \\
\hline & & $\underline{\mathrm{R}}$ & $\begin{array}{l}\text { A force not parallel to } \mathbf{z} \text {, orthogonal to the directions of two } \underline{P} \text {-joints and crossing the } \\
\text { axis of the unactuated } \mathrm{R} \text {-joint }\end{array}$ \\
\hline & \multirow[t]{2}{*}{ Permutation of PPPR } & $\underline{P}$ & $\begin{array}{l}\text { A force orthogonal to the directions of two } \underline{P} \text {-joints and crossing the axis of the } \\
\text { unactuated } \mathrm{R} \text {-joint }\end{array}$ \\
\hline & & $\underline{\mathrm{R}}$ & A moment corresponding to a line at infinity that does not pass through point $\underline{j}=(\mathbf{z}, 0)$ \\
\hline \multirow[t]{22}{*}{5} & \multirow[t]{2}{*}{ Permutation of R̀̀ंR̀ŔŔ } & $\underline{\dot{R}}$ & $\begin{array}{l}\text { A force collinear with the intersection line of two planes: the first containing the axes of } \\
\text { two R-joints and the second containing the axes of two unactuated R-joints }\end{array}$ \\
\hline & & $\underline{\underline{R}}$ & A force parallel to $\mathbf{z}$ and intersecting the axis of unactuated $\mathbf{R}_{\text {-joint }}$ \\
\hline & \multirow[t]{2}{*}{ Permutation of R̀RŔŔŔ } & $\underline{\dot{R}}$ & A force parallel to $\mathbf{m}_{i}$ and intersecting the axis of the unactuated $\mathrm{R}$-joint \\
\hline & & $\underline{R}$ & $\begin{array}{l}\text { A force collinear with the intersection line of two planes: one containing the axes of two } \\
\text { R-joints and the second one containing the axes of two unactuated R-joints }\end{array}$ \\
\hline & \multirow{2}{*}{$\begin{array}{l}\text { Permutation of PR̀R̀̀ंŔ } \\
\text { (the P-joint being }\end{array}$} & $\underline{P}$ & A force parallel to $\mathbf{z}$ and intersecting the axis of the $\mathrm{R}$-joint \\
\hline & & $\underline{\mathrm{R}}$ & $\begin{array}{l}\text { The determination of the actuation wrench requires further details on the geometry of } \\
\text { the limb }\end{array}$ \\
\hline & along $\mathbf{z})$ & $\underline{\mathrm{R}}$ & A force directed along $\mathbf{z}$ \\
\hline & \multirow[t]{3}{*}{ Permutation of PR̀RŔŔ } & $\underline{P}$ & $\begin{array}{l}\text { A force collinear with the intersection line of two planes: the first containing the axes of } \\
\text { two R-joints and the second containing the axes of two R-joints }\end{array}$ \\
\hline & & $\underline{\underline{\mathrm{R}}}$ & \multirow{2}{*}{$\begin{array}{l}\text { The determination of the actuation wrench requires further details on the geometry } \\
\text { of the limb }\end{array}$} \\
\hline & & $\underline{\mathrm{R}}$ & \\
\hline & \multirow{3}{*}{$\begin{array}{l}\text { Permutation of PRŔŔŔ } \\
\text { (the P-joint being } \\
\text { along } \mathbf{m}_{i} \text { ) }\end{array}$} & $\underline{P}$ & A force parallel to $\mathbf{m}_{i}$ and intersecting the axis of the $\dot{R}_{\text {-joint }}$ \\
\hline & & $\underline{\underline{\mathrm{R}}}$ & A force parallel to $\mathbf{m}_{i}$ \\
\hline & & $\underline{\underline{R}}$ & \multirow{8}{*}{$\begin{array}{l}\text { The determination of the actuation wrench requires further details on the geometry } \\
\text { of the limb }\end{array}$} \\
\hline & \multirow[t]{3}{*}{ Permutation of PPR̀RR } & $\underline{P}$ & \\
\hline & & $\underline{\mathrm{R}}$ & \\
\hline & & $\underline{R}$ & \\
\hline & \multirow[t]{3}{*}{ Permutation of PPRŔŔ } & $\underline{P}$ & \\
\hline & & $\underline{\mathrm{R}}$ & \\
\hline & & $\underline{R}$ & \\
\hline & \multirow[t]{3}{*}{ Permutation of PPPRŔ } & $\underline{P}$ & \\
\hline & & $\underline{\dot{R}}$ & A moment corresponding to a line at infinity that does not pass through point $\underline{j}=(\mathbf{z}, 0)$ \\
\hline & & $\underline{\mathrm{R}}$ & A moment corresponding to a line at infinity that does not pass through point $\underline{\mathrm{m}}=\left(\mathbf{m}_{i}, 0\right)$ \\
\hline
\end{tabular}


R5 If a basis of $\mathrm{W}^{a}$ is composed of four actuation forces, then it can contain at most one pair of parallel actuation forces, provided that their plane does not contain the unit vector $\mathbf{z}$.

Proof Clearly, if a basis of $\mathrm{W}^{a}$ contains more than one pair of parallel actuation forces, then one can compute another basis of $\mathrm{W}^{a}$ with more than one actuation moment, and therefore, rule $\mathbf{R} \mathbf{1}$ will not be satisfied.

R6 If a basis of $\mathrm{W}^{a}$ is composed of four actuation forces where two forces $\hat{F}_{1}$ and $\hat{F}_{2}$ are parallel, then the plane containing these forces must not contain the unit vector $\mathbf{z}$.

Proof If the plane containing the two parallel actuation forces contains the unit vector $\mathbf{z}$, it follows that the two actuation forces $\hat{F}_{1}$ and $\hat{F}_{2}$ and the two constraint moments $\hat{M}_{c 1}$ and $\hat{M}_{c 2}$ will belong altogether to the union of two flat pencils of lines. Accordingly, $\operatorname{dim}\left(\operatorname{span}\left(\hat{F}_{1}, \hat{F}_{2}, \hat{M}_{c 1}, \hat{M}_{c 2}\right)\right) \leq 3$ and the dimension of $\mathrm{w}^{a}+\mathrm{W}^{c}$ will be lower than or equal to 5 in any robot configuration.

R7 If a basis of $\mathrm{w}^{a}$ is composed of four actuation forces, then at most three of these forces can be coplanar.

Proof Indeed, if the four forces are coplanar, then the actuation wrench system fails to be a 4-system in a general configuration, since four coplanar forces can span at most a 3-system.

$\mathbf{R 8}$ A basis of $\mathrm{W}^{a}$ can contain at most one actuation force parallel to $\mathbf{z}$.

Proof Indeed, if two actuation forces, $\hat{F}_{1}$ and $\hat{F}_{2}$, are directed along $\mathbf{z}$, then $\operatorname{span}\left(\hat{F}_{1}, \hat{F}_{2}\right)$ is equivalent to $\operatorname{span}\left(\hat{F}_{1}, \hat{M}_{12}\right)$ where $\hat{M}_{12}$ corresponds to a line at infinity that necessarily passes through point $\mathrm{j}=(\mathbf{z}, 0)$. Accordingly, in that case, rule $\mathbf{R} 2$ will not be satisfied.

R9 If a basis of $\mathrm{W}^{a}$ is composed of four actuation forces, these forces cannot be orthogonal to a common direction.

Proof Indeed, if the four actuation forces are (in a general configuration) orthogonal to a common direction, then they will intersect a common line at infinity, which necessarily intersects all the wrenches of the constraint wrench system. Therefore, in that case, the four actuation forces plus the $2-\$ \infty$-system of constraint wrenches form a singular complex.

\section{Typical Classes of SMGs}

Based on the properties presented in the previous section, two main classes of 3T1R PMs can be identified. For PMs of the first class, the limbs apply, in a general configuration, one actuation moment and three actuation forces on the moving platform. On the other hand, the actuation wrenches applied by the limbs of PMs of the second class in a general configuration are pure forces.

The first class contains only one case. However, by following rules R1... R9, some sub-classes of the second class can be obtained.

In this section, the wrench graphs corresponding to the first class and to the five sub-classes of the second class will be represented. It is noteworthy that the sub-classes are defined by the types of the actuation wrenches applied by the limbs and the geometric relations between these wrenches, the constraint wrench system being the same for any 3T1R PM.

\subsection{Class A: One pure actuation moment is applied by the limbs.}

Class A.1 One actuation moment plus three actuation forces. In that case, the three actuation forces cannot be coplanar and none of these forces can be parallel to another.

The corresponding wrench graph is given in Table 3.

\subsection{Class B: The actuation wrenches applied by the limbs are pure forces.}

Class B.1 Two parallel actuation forces plus two other actuation forces;

Class B.2 Three coplanar actuation forces plus a fourth actuation force that does not belong to the plane of the first three ones.

Class B.3 Four actuation forces that are mutually skew where none of the four forces is parallel to $\mathbf{z}$;

Class B.4 Four actuation forces that are mutually skew where one of the four forces is parallel to $\mathbf{z}$;

Class B.5 Two pairs of concurrent actuation forces.

A wrench graph for each sub-class is given in Table 3.

\subsection{Superbracket Decomposition}

\section{A.1- One actuation moment plus three actuation forces}

Let us consider a PM of class A.1. A basis of the actuation wrench system $\mathrm{W}^{a}$ of the PM can be composed of three actuation forces $\hat{F}_{1}, \hat{F}_{2}$ and $\hat{F}_{3}$ and one actuation moment $\hat{M}_{a}$. Now let $\hat{M}_{c 1}$ and $\hat{M}_{c 2}$ be two constraint moments that form a basis of the constraint wrench system $\mathrm{W}^{c}$ of the PM. Accordingly, the overall Jacobian matrix of this sub-class of 
Table 3. Wrench Graph and Superbracket Decomposition for SMGs of Classes A and B.

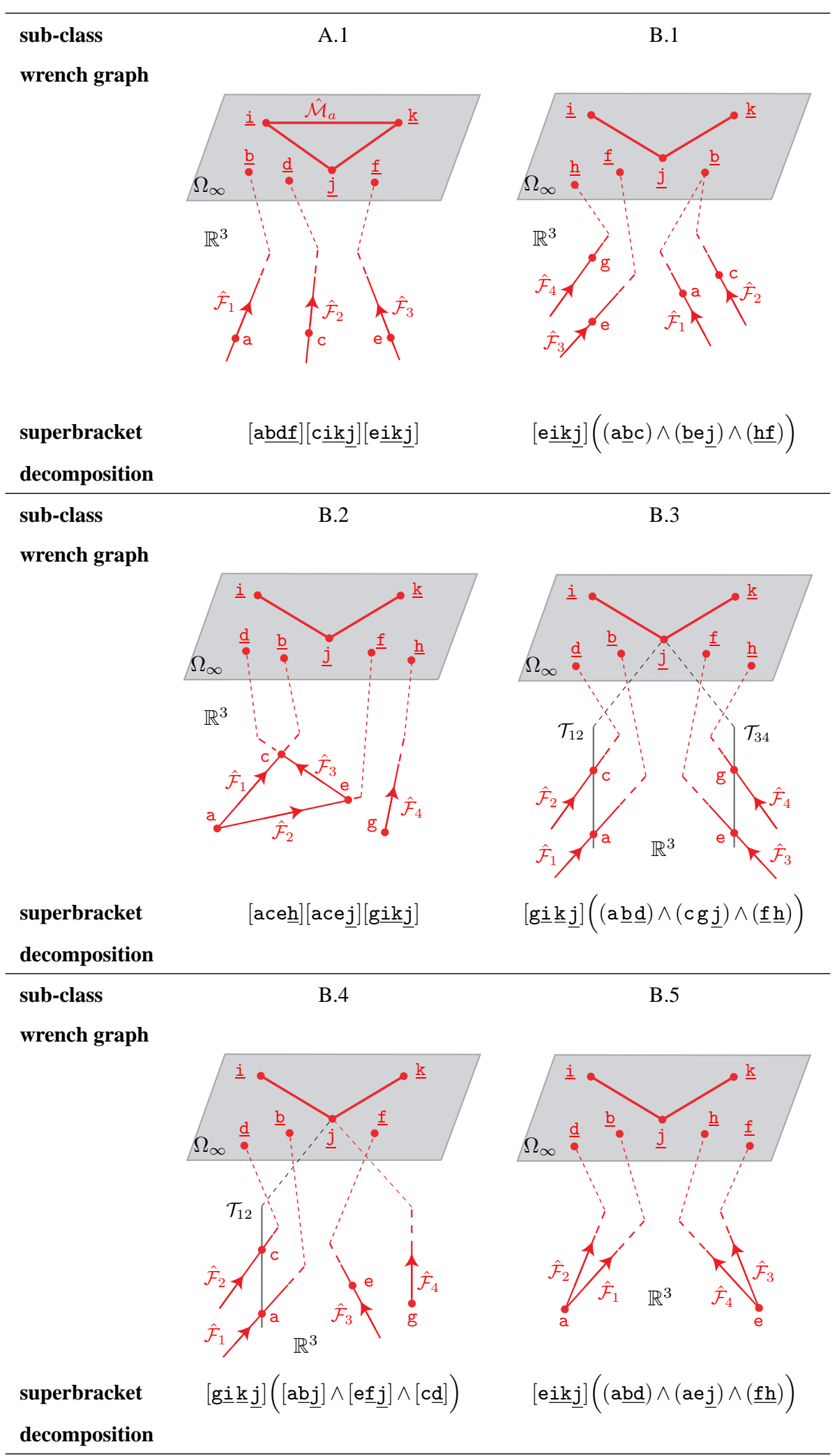


SMGs takes the form:

$$
\mathbf{J}^{T}=\left[\hat{F}_{1} \hat{F}_{2} \hat{F}_{3} \hat{M}_{a} \hat{M}_{c 1} \hat{M}_{c 2}\right]
$$

From rule R1, the actuation moment $\hat{M}_{a}$ must correspond to a line at infinity that does not pass through point $\underline{j}$, in a general configuration of the PM. Moreover, in a general configuration, the three actuation forces cannot be coplanar and none of these forces can be parallel to another. Let us consider the general case satisfying these conditions by considering that the three actuation forces are skew (in a general configuration). It is known that three moments are always mutually concurrent. Therefore, the six wrenches composing $\mathbf{J}$ can be written as: $\hat{F}_{1}=\left(\mathbf{f}_{1}, \mathbf{r}_{A_{1}} \times \mathbf{f}_{1}\right)=$ ab $\hat{F}_{2}=\left(\mathbf{f}_{2}, \mathbf{r}_{A_{2}} \times \mathbf{f}_{2}\right)=\mathrm{c} \underline{\mathrm{d}}, \hat{F}_{3}=\left(\mathbf{f}_{3}, \mathbf{r}_{A_{3}} \times \mathbf{f}_{3}\right)=\mathrm{e} \underline{\underline{f}}, \hat{M}_{a}=\left(\mathbf{0}, \mathbf{m}_{a}\right)=\underline{\mathrm{ik}}, \hat{M}_{c 1}=\underline{\mathrm{i}} \underline{\mathrm{j}}=\left(\mathbf{0}, \mathbf{m}_{c 1}\right) \hat{M}_{c 2}=\underline{\mathrm{k}} \underline{\mathrm{j}}=\left(\mathbf{0}, \mathbf{m}_{c 2}\right)$ The corresponding superbracket is then expressed and simplified as follows:

$$
S=[a \underline{b} \mathrm{~cd} \text { ef } \underline{i k} \underline{i} \underline{j} \underline{k j}]=[a \underline{b d f}][c \underline{i k} \underline{j}][e \underline{i k} \underline{j}]
$$

Such a PM has two singularity conditions:

(a) $\left(\mathbf{f}_{1} \times \mathbf{f}_{2}\right) \cdot \mathbf{f}_{3}=0$

(b) $\left(\mathbf{m}_{c 1} \times \mathbf{m}_{c 2}\right) \cdot \mathbf{m}_{a}=0$

B.1- Two parallel actuation forces plus two other skew actuation forces

The overall Jacobian matrix of this sub-class of SMGs takes the form:

$$
\mathbf{J}^{T}=\left[\hat{F}_{1} \hat{F}_{2} \hat{F}_{3} \hat{F}_{4} \hat{M}_{c 1} \hat{M}_{c 2}\right]
$$

where $\hat{F}_{1}$ is parallel to $\hat{F}_{2}$. Let $\hat{F}_{1}=\left(\mathbf{f}_{1}, \mathbf{r}_{A_{1}} \times \mathbf{f}_{1}\right)=\mathrm{ab}, \hat{F}_{2}=\left(\mathbf{f}_{1}, \mathbf{r}_{A_{2}} \times \mathbf{f}_{1}\right)=\mathrm{cb}, \hat{F}_{3}=\left(\mathbf{f}_{3}, \mathbf{r}_{A_{3}} \times \mathbf{f}_{3}\right)=$ ef and $\hat{F}_{4}=$ $\left(\mathbf{f}_{4}, \mathbf{r}_{A_{4}} \times \mathbf{f}_{4}\right)=$ gh be the four actuation forces. In turn, let $\hat{M}_{c 1}=\left(\mathbf{0}, \mathbf{m}_{c 1}\right)=\underline{i \mathrm{j}}$ and $\hat{M}_{c 2}=\left(\mathbf{0}, \mathbf{m}_{c 2}\right)=\underline{\mathrm{kj}}$ be the two constraint moments.

The corresponding superbracket is expressed and decomposed as:

$$
\begin{aligned}
& S=[\mathrm{ab} \underline{\mathrm{c}} \underline{\mathrm{b}} \text { ef } \mathrm{g} \underline{\mathrm{h}} \underline{\mathrm{i}} \underline{\mathrm{j}} \underline{\mathrm{k}} \underline{\mathrm{j}}] \\
& =[\mathrm{e} \underline{i k} \underline{j}]([a \underline{b} c \underline{h}][\underline{b} e \underline{f} \underline{j}]-[a \underline{b} c \underline{f}][\underline{b} e \underline{h} \underline{j}]) \\
& =[\mathrm{e} \underline{i k} \underline{j}]([a \underline{b} \mathrm{c} \underline{\hat{h}}][\underline{b} e \underline{\dot{j}} \underline{j}]) \\
& =[e \underline{i k} \underline{j}]((a \underline{b} c) \wedge(\underline{b} e \underline{j}) \wedge(\underline{h f}))
\end{aligned}
$$

Let $\mathbf{u}_{a c}$ be the unit vector of the finite line joining the projective points a and c. This sub-class of SMGs has two main singularity conditions:

(a) $\left(\mathbf{m}_{c 1} \times \mathbf{m}_{c 2}\right) \cdot \mathbf{z}=0$. Clearly, this is the condition for constraint singularities.

(b) $\left(\left(\mathbf{f}_{1} \times \mathbf{u}_{a c}\right) \times\left(\mathbf{f}_{1} \times \mathbf{z}\right)\right) \cdot\left(\mathbf{f}_{4} \times \mathbf{f}_{3}\right)=0 \Rightarrow$

1. $\mathbf{f}_{1} \| \mathbf{u}_{\mathrm{ac}}$

2. $\mathbf{f}_{1} \| \mathbf{z}$

3. $\mathbf{f}_{3} \| \mathbf{f}_{4}$.

B.2- Three coplanar actuation forces plus a fourth actuation force

The overall Jacobian matrix of this sub-class of SMGs takes the form:

$$
\mathbf{J}^{T}=\left[\hat{F}_{1} \hat{F}_{2} \hat{F}_{3} \hat{F}_{4} \hat{M}_{c 1} \hat{M}_{c 2}\right]
$$

where $\hat{F}_{1} \hat{F}_{2}$ and $\hat{F}_{3}$ intersect each one another at a finite point. Let $\hat{F}_{1}=$ ac, $\hat{F}_{2}=$ ae, $\hat{F}_{3}=$ ce and $\hat{F}_{4}=$ gh be the four actuation forces. In turn, let $\hat{M}_{c 1}=\underline{i} \mathrm{j}$ and $\hat{M}_{c 2}=\underline{\mathrm{kj}}$ be the two constraint moments. 
The corresponding superbracket is expressed and developed as follows:

$$
S=[\operatorname{ac} \text { ae ce gh } \underline{i} \underline{j} \underline{k} \underline{j}]=[\operatorname{ace} \underline{h}][\operatorname{ace} \underline{j}][\operatorname{gik} \underline{j}]
$$

This sub-class of SMGs has three singularity conditions:

(a) $\left(\mathbf{m}_{c 1} \times \mathbf{m}_{c 2}\right) \cdot \mathbf{z}=0$

(b) plane (ace) $\| \mathbf{f}_{4}$

(c) plane (ace) $\| \mathbf{z}$

\section{B.3- Four skew lines none of which being directed along $z$}

The overall Jacobian matrix of this sub-class of SMGs takes the form:

$$
\mathbf{J}^{T}=\left[\hat{F}_{1} \hat{F}_{2} \hat{F}_{3} \hat{F}_{4} \hat{M}_{c 1} \hat{M}_{c 2}\right]
$$

where $\hat{F}_{1} \hat{F}_{2}, \hat{F}_{3}$ and $\hat{F}_{4}$ correspond to four finite lines that are mutually skew and none of them is directed along $\mathbf{z}$. There exists (in a general configuration) a finite line $\mathcal{L}_{i j}$ parallel to $\mathbf{z}$ that crosses any couple of actuation forces $\hat{F}_{i}$ and $\hat{F}_{j}$. Let $\hat{F}_{1}=\left(\mathbf{f}_{1}, \mathbf{r}_{1} \times \mathbf{f}_{1}\right), \hat{F}_{2}=\left(\mathbf{f}_{2}, \mathbf{r}_{2} \times \mathbf{f}_{2}\right), \hat{F}_{3}=\left(\mathbf{f}_{3}, \mathbf{r}_{3} \times \mathbf{f}_{3}\right)$ and $\hat{F}_{4}=\left(\mathbf{f}_{4}, \mathbf{r}_{4} \times \mathbf{f}_{4}\right)$ be the four actuation forces. In turn, let $\hat{M}_{c 1}=\underline{\mathrm{i}} \underline{\mathrm{j}}$ and $\hat{M}_{c 2}=\underline{\mathrm{k} j}$ be the two constraint moments. The superbracket then is depicted and developed as:

$$
\begin{aligned}
& S=[\mathrm{ab} \mathrm{c} \underline{\mathrm{d}} \text { ef } \mathrm{g} \underline{\mathrm{h}} \underline{\mathrm{i}} \underline{\mathrm{j}} \underline{\mathrm{k}} \mathrm{j}] \\
& =[\mathrm{g} \underline{\mathrm{ik}} \underline{j}]([\mathrm{abdf}][\operatorname{cg} \underline{\hat{h}} \underline{j}]) \\
& =[\mathrm{g} \underline{i k j}]((a \underline{a d}) \wedge(\mathrm{cg} \underline{j}) \wedge(\underline{f h}))
\end{aligned}
$$

This sub-class of SMGs has two main singularity conditions:

(a) $\left(\mathbf{m}_{c 1} \times \mathbf{m}_{c 2}\right) \cdot \mathbf{z}=0$.

(b) $\left(\left(\mathbf{z} \times \mathbf{u}_{c g}\right) \times\left(\mathbf{f}_{1} \times \mathbf{f}_{2}\right)\right) \cdot\left(\mathbf{f}_{4} \times \mathbf{f}_{3}\right)=0$

where $\mathbf{u}_{c g}$ is the unit vector of a finite line non-parallel to $\mathbf{z}$ and crossing lines $\mathcal{L}_{12}$ and $\mathcal{L}_{34}$.

\section{B.4- Four skew lines one of which being directed along $z$}

The overall Jacobian matrix of this sub-class of SMGs takes the form:

$$
\mathbf{J}^{T}=\left[\hat{F}_{1} \hat{F}_{2} \hat{F}_{3} \hat{F}_{4} \hat{M}_{c 1} \hat{M}_{c 2}\right]
$$

where $\hat{F}_{1} \hat{F}_{2}, \hat{F}_{3}$ and $\hat{F}_{4}$ correspond to four finite lines that are mutually skew and $\hat{F}_{4}$ is parallel to z. We know that, in a general configuration, there exists a finite line $\mathcal{L}_{i j}$ parallel to $\mathbf{z}$ that crosses any couple of actuation forces among $\hat{F}_{1}, \hat{F}_{2}$ and $\hat{F}_{3}$. Therefore, let $\mathcal{L}_{12} \| \mathbf{z}=$ ac be the finite line parallel to $\mathbf{z}$ that crosses, in a general configuration, $\hat{F}_{1}$ and $\hat{F}_{2}$ at the finite points a and c, respectively. Therefore, points a, $c$ and $\underline{j}$ are aligned.

Let $\hat{F}_{1}=\mathrm{ab}, \hat{F}_{2}=\mathrm{c} \underline{\mathrm{d}}, \hat{F}_{3}=\mathrm{e} \underline{\mathrm{f}}$ and $\hat{F}_{4}=\mathrm{g} \underline{\mathrm{j}}$ be the four actuation forces. In turn, let $\hat{M}_{c 1}=\underline{\mathrm{i}} \underline{\mathrm{j}}$ and $\hat{M}_{c 2}=\underline{\mathrm{k}} \underline{\mathrm{j}}$ be the two constraint moments.

The corresponding superbracket is expressed and simplified as follows:

$$
\begin{aligned}
& S=[\mathrm{ab} c \underline{\mathrm{d}} \text { ef } \mathrm{g} \underline{\mathrm{j}} \underline{\mathrm{i}} \underline{\mathrm{j}} \underline{\mathrm{k}} \underline{\mathrm{j}}]
\end{aligned}
$$

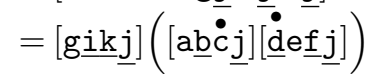

$$
\begin{aligned}
& =[\mathrm{g} \underline{i k} \underline{j}]((a \underline{b} \underline{j}) \wedge(e \underline{f} \underline{j}) \wedge(c \underline{d}))
\end{aligned}
$$

This sub-class of SMGs has two singularity conditions: 
(a) $\left(\mathbf{m}_{c 1} \times \mathbf{m}_{c 2}\right) \cdot \mathbf{z}=0$.

(b) $\left(\left(\mathbf{f}_{1} \times \mathbf{z}\right) \times\left(\mathbf{f}_{3} \times \mathbf{z}\right)\right) \cdot \mathbf{f}_{2}=0$

\section{B.5- Two pairs of intersecting actuation forces}

The overall Jacobian matrix of this sub-class of SMGs has the form:

$$
\mathbf{J}^{T}=\left[\hat{F}_{1} \hat{F}_{2} \hat{F}_{3} \hat{F}_{4} \hat{M}_{c 1} \hat{M}_{c 2}\right]
$$

where $\hat{F}_{1}=\left(\mathbf{f}_{1}, \mathbf{r}_{1} \times \mathbf{f}_{1}\right)=\mathrm{ab}, \hat{F}_{2}=\left(\mathbf{f}_{2}, \mathbf{r}_{1} \times \mathbf{f}_{2}\right)=\mathrm{ad}, \hat{F}_{3}=\left(\mathbf{f}_{3}, \mathbf{r}_{3} \times \mathbf{f}_{3}\right)=\mathrm{eq}, \hat{F}_{4}=\left(\mathbf{f}_{4}, \mathbf{r}_{4} \times \mathbf{f}_{4}\right)=\mathrm{eh}, \hat{M}_{c 1}=\underline{i j}$ and $\hat{M}_{c 2}=\underline{\mathrm{k}} \mathbf{j}$.

The corresponding superbracket is expressed and simplified as follows:

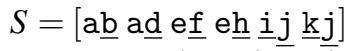

$$
\begin{aligned}
& =[\mathrm{e} \underline{\mathrm{ik}} \underline{j}]([\mathrm{abdf}][\mathrm{ae} \underline{\underline{h}} \underline{j}]) \\
& =[e \underline{i k j} \underline{j}]((a \underline{b d}) \wedge(a e \underline{j}) \wedge(\underline{f h}))
\end{aligned}
$$

This sub-class of SMGs has two main singularity conditions:

(a) $\left(\mathbf{m}_{c 1} \times \mathbf{m}_{c 2}\right) \cdot \mathbf{z}=0$.

(b) $\left(\left(\mathbf{z} \times \mathbf{u}_{a e}\right) \times\left(\mathbf{f}_{1} \times \mathbf{f}_{2}\right)\right) \cdot\left(\mathbf{f}_{4} \times \mathbf{f}_{3}\right)=0$

where $\mathbf{u}_{\mathrm{ae}}$ is the unit vector of the finite line joining points a and $\mathrm{e}$.

\section{Applications}

\subsection{The Quadrupteron PM (Class B.1)}

The Quadrupteron PM [5] illustrated in Fig. 2, is composed of three identical limbs: $\mathcal{L}_{i}=\underline{\mathrm{P}}^{i} \overline{\mathrm{R}}_{1}^{i} \overline{\mathrm{R}}_{2}^{i} \overline{\mathrm{R}}_{3}^{i} \grave{\mathrm{R}}^{i}, i=1,2$, 3, plus one limb $\mathcal{L}_{4}=\underline{\mathrm{P}}^{4} \dot{\mathrm{R}}_{1}^{4} \dot{\mathrm{R}}_{2}^{4} \dot{\mathrm{R}}_{3}^{4}$.

The actuated joint $\underline{\mathrm{P}}^{i}$ of the $i$ th $\operatorname{limb}(i=1,2,3)$ is directed along $\mathbf{m}_{i}$ which is parallel to the axes of the limb's three $\overline{\mathrm{R}}^{i}$-joints such that $\mathbf{m}_{1}\left\|\mathbf{m}_{3}\right\| \mathbf{x}$ and $\mathbf{m}_{2} \| \mathbf{y}$. In turn, the actuated joint $\underline{P}^{4}$ of the fourth limb is directed along $\mathbf{z}$ which is parallel to the axes of the limb's three $\dot{R}^{i}$-joints. It is noteworthy that vectors $\mathbf{x}, \mathbf{y}$ and $\mathbf{z}$ have fixed directions.

Limb $\mathcal{L}_{4}$ is a 4-dof limb of Table 1. This limb is sufficient to make the Quadrupteron PM free of constraint singularities.

From Table 2, it follows that the four limbs of the Quadrupteron PM apply four actuation forces $\hat{F}_{1}=\left(\mathbf{x}, \mathbf{r}_{A_{1}} \times \mathbf{x}\right)$, $\hat{F}_{2}=\left(\mathbf{y}, \mathbf{r}_{A_{2}} \times \mathbf{y}\right), \hat{F}_{3}=\left(\mathbf{x}, \mathbf{r}_{A_{3}} \times \mathbf{x}\right)$ and $\hat{F}_{4}=\left(\mathbf{z}, \mathbf{r}_{A_{4}} \times \mathbf{z}\right)$. According to class B.1, the Quadrupteron PM exhibits an actuation singularity whenever:

1. $\mathbf{f}_{1} \| \mathbf{u}_{\mathrm{ac}}$

2. $\mathbf{f}_{1} \| \mathbf{z}$

3. $\mathbf{f}_{3} \| \mathbf{f}_{4}$

For the Quadrupteron PM, $\mathbf{f}_{1} \equiv \mathbf{f}_{3} \equiv \mathbf{x}, \mathbf{f}_{2} \equiv \mathbf{y}, \mathbf{f}_{4} \equiv \mathbf{z}$ and $\mathbf{u}_{\mathrm{ac}}$ is the unit vector of line $A_{1} A_{3}$. As a result, the second and the third singularity conditions are impossible. Consequently, the only possible singularity condition is: $\left(A_{1} A_{3} \| \mathbf{x}\right)$, which is illustrated in Fig. 3.

\subsection{A 2-PRRU-PRRR-PRPP PM (class A.1)}

Now let us modify the architecture of the Quadrupteron PM in order to obtain a PM of the sub-class A.1. Accordingly, we should replace the third limb $\mathcal{L}_{3}$ of the Quadrupteron PM with a limb generating an actuation moment. From Table 2, the limb PRPP satisfies the required condition. As a result, the 2-PRRU-PRRR-PRPP PM, shown in Fig. 4, is obtained. It is noteworthy that this architecture is introduced as an example of the sub-class A.1. However, compared to the original Quadrupteron PM, the 2-PRRU-PRRR-P $\underline{R} P P$ PM is less interesting regarding the stiffness and accuracy.

It can be noticed that the proposed PM is free of constraint singularities. The condition for actuation singularities is given by: $\left(\mathbf{f}_{1} \times \mathbf{f}_{2}\right) \cdot \mathbf{f}_{3}=0$. Since, for the proposed 2-PRRU-PRRR-PRPP PM, $\mathbf{f}_{1} \equiv \mathbf{x}, \mathbf{f}_{2} \equiv \mathbf{y}$ and $\mathbf{f}_{3} \equiv \mathbf{z}$, this PM is also free of actuation singularities. 


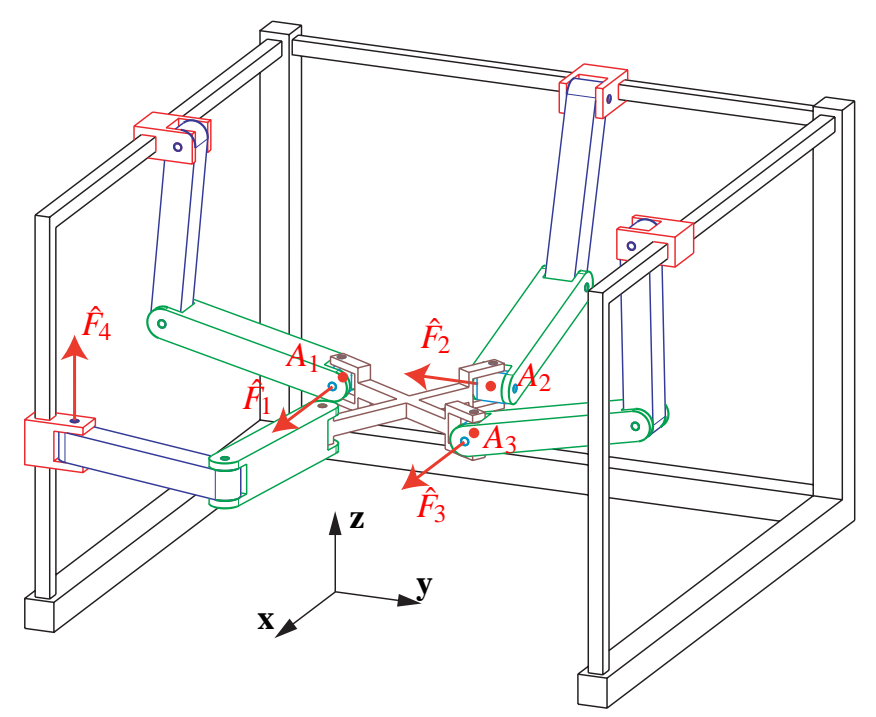

Fig. 2. The Quadrupteron PM.

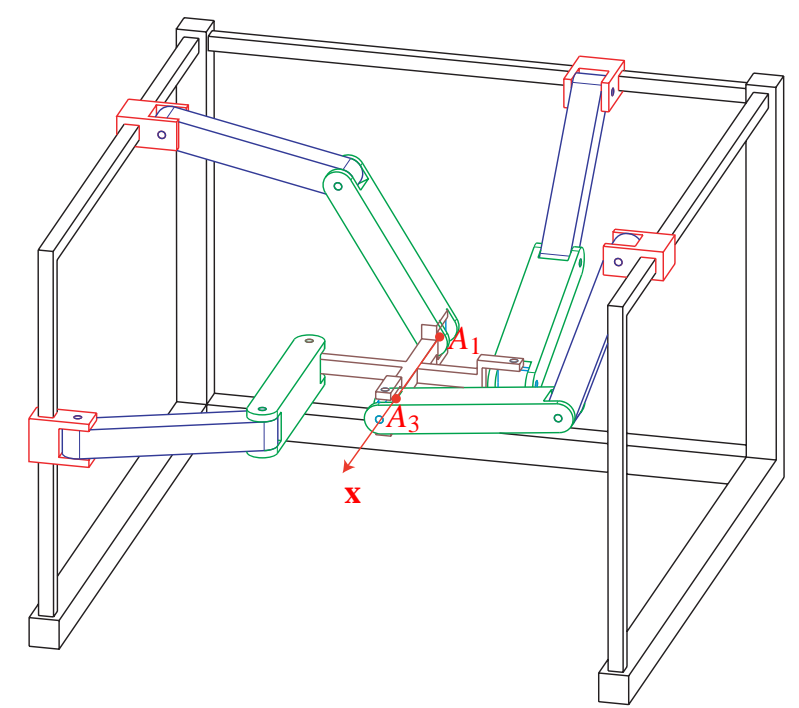

Fig. 3. Acutation Singularity of the Quadrupteron PM.

\subsection{The 3-URU-SPS PM (class B.2)}

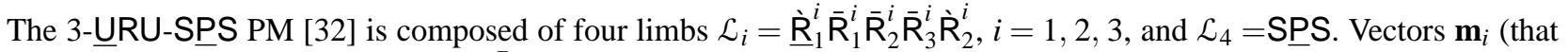
correspond to the directions of the limb's $\overline{\mathrm{R}}$-joints) are parallel to the horizontal plane (xOy).

From Table 2, the actuation force of the $i$ th $\operatorname{limb}(i=1,2,3)$ is parallel to plane $(\mathbf{x} O \mathbf{y})$. Therefore, from rule $\mathbf{R . 9}$, we cannot obtain a valid 3T1R PM with four limbs of this type.

The actuation force of limb $\mathcal{L}_{4}=\mathrm{SPS}$ is directed along the actuated P-joint. The actuation forces $\hat{F}_{i}$ of limbs $\mathcal{L}_{i}$ $(i=1,2,3)$ can be selected to be coplanar, as shown in Fig. 5 .

A constraint singularity of the 3-URU-SPS PM occurs if the three constraint moments $\hat{M}_{c i}=\left(\mathbf{0}, \mathbf{m}_{i} \times \mathbf{z}\right), i=1,2,3$, applied by the three limbs $\mathcal{L}_{1}, \mathcal{L}_{2}$ and $\mathcal{L}_{3}$ form a $c \leq 1$-system. Therefore, the constraint singularities correspond to configurations in which the three vectors $\mathbf{m}_{i}, i=1,2,3$, become parallel.

Let $\hat{F}_{1}=$ ac, $\hat{F}_{2}=$ ae, $\hat{F}_{3}=$ ce and $\hat{F}_{4}=$ gh. From Sec. 3.1, the 3-URU-SPS PM exhibits an actuation singularity whenever:

1. $[$ aceh $]=0$. This occurs if plane ace degenerates or if the actuation force $\hat{F}_{4}$ is parallel to plane ace.

2. $[a c e j]=0$. This occurs if plane ace degenerates or if $\mathbf{z}$ is parallel to plane ace. The latter condition cannot occur as long as the PM is in the 3T1R motion mode since in this mode, plane ace is parallel to plane $(\mathbf{x} O \mathbf{y})$. 


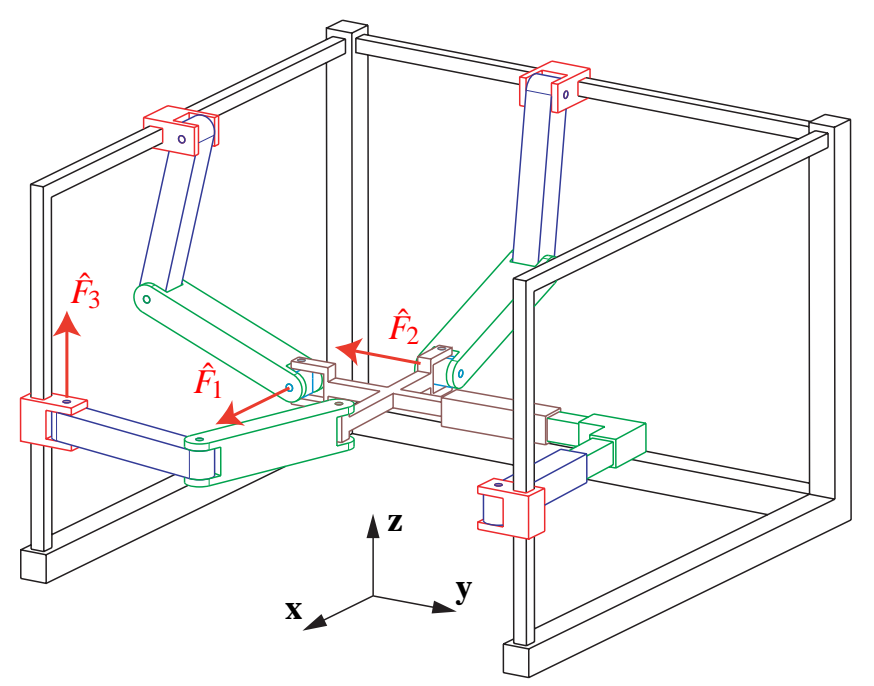

Fig. 4. A 2-PRRU-PRRR-PRPP PM.

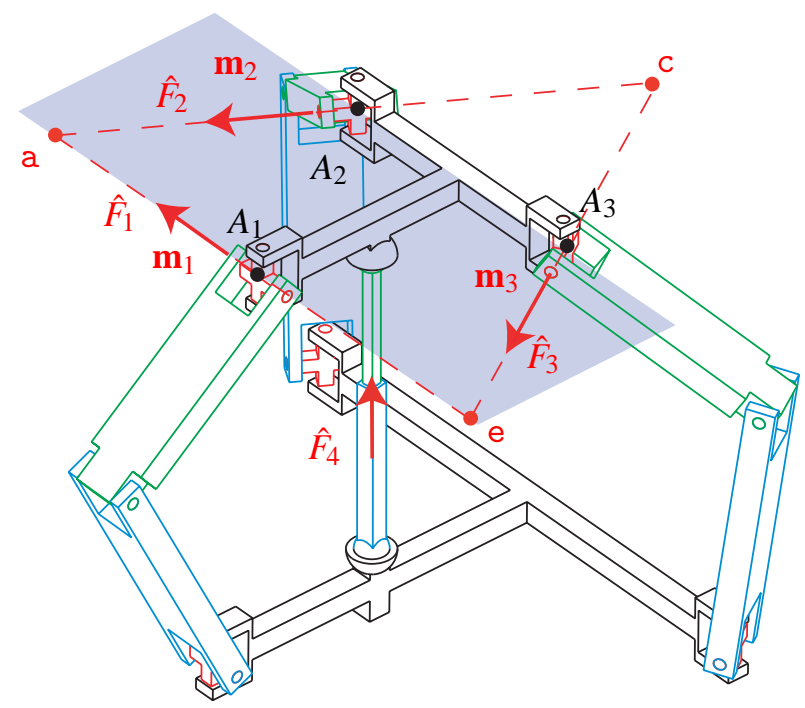

Fig. 5. The 3-ㅂRU-SP्S PM.

\subsection{The 4-UPU PM (class B.3)}

The 4-UPU PM [32], shown in Fig. 6 is composed of four limbs $\mathcal{L}_{i}=\dot{\mathrm{R}}_{1}^{i} \overline{\mathrm{R}}_{1}^{i} \underline{\mathrm{P}} \overline{\mathrm{R}}_{2}^{i} \grave{\mathrm{R}}_{2}^{i}, i=1, \ldots, 4$. Vectors $\mathbf{m}_{i}$ (that correspond to the directions of the limb's $\overline{\mathrm{R}}$-joints) are parallel to the horizontal plane $(\mathbf{x} O \mathbf{y})$.

The axes of the first and the second (respectively of the third and the fourth) revolute joints of the $i$ th limb are arranged with intersecting and perpendicular axes and are thus assimilated to a $\mathrm{U}$-joint centered at point $A_{i}$ (respectively at point $B_{i}$ ). Therefore, the actuation force of the $i$ th limb is collinear with line $A_{i} B_{i}$. Let $\hat{F}_{i}=\left(\mathbf{f}_{i}, \mathbf{r}_{B_{i}} \times \mathbf{f}_{i}\right)$ denote the actuation force of the $i$ th limb.

The $i$ th limb applies one constraint moment $\hat{M}_{i}=\left(\mathbf{0}, \mathbf{m}_{i} \times \mathbf{z}\right)$. In a general configuration, the four actuation forces applied by the four limbs of the 4-UPU PM correspond to four skew finite lines. Therefore, according to Sec. 3.1, the 4-UPU PM exhibits:

- a constraint singularity whenever the four vectors $\mathbf{m}_{i}$ are parallel;

- an actuation singularity whenever

$$
\left(\left(\mathbf{f}_{i} \times \mathbf{f}_{j}\right) \times\left(\mathbf{f}_{k} \times \mathbf{f}_{l}\right)\right) \cdot\left(\mathbf{u}_{i j}^{k l} \times \mathbf{z}\right)=0
$$

where $(i, j, k, l)$, is a permutation of $(1,2,3,4)$ and vector $\mathbf{u}_{i j}^{k l}$ is defined in Sec. 3.1. 


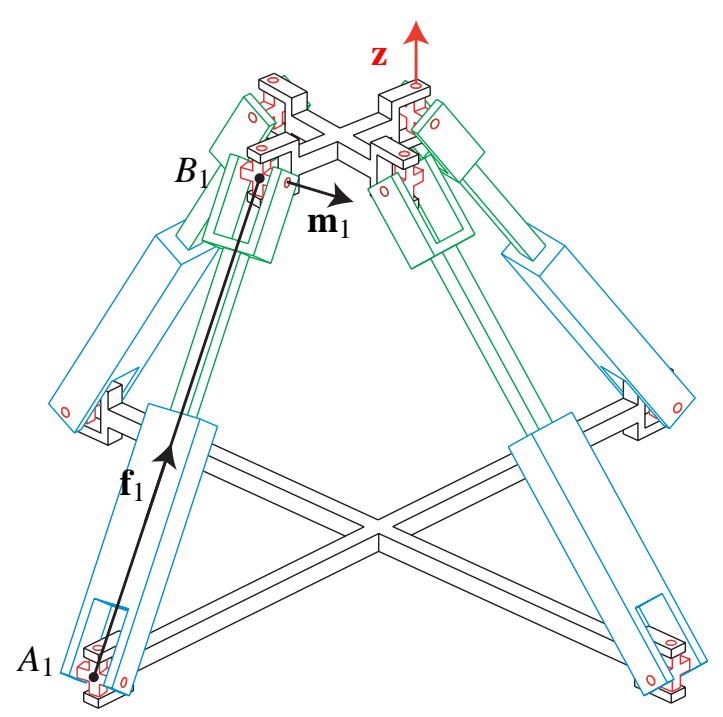

Fig. 6. The 4-UPU PM.

\subsection{The 3-UPU-PRRR PM (class B.4)}

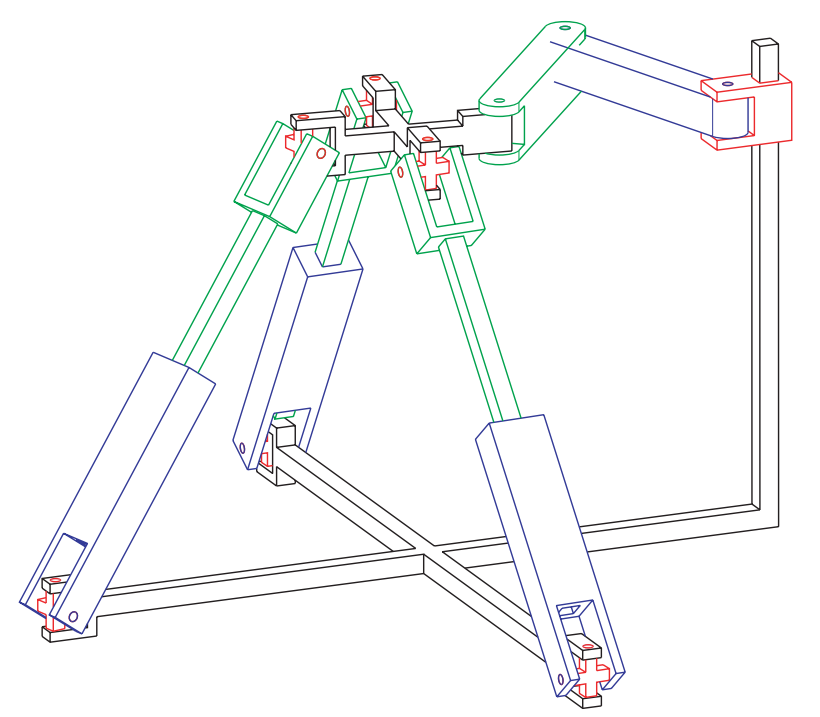

Fig. 7. The 3-UPU-PRRR PM.

Now let us modify the architecture of the 4-UPU PM in order to obtain a PM of the sub-class B.4. Accordingly, we should replace one of the four UPU limbs with a limb applying an actuation force along $\mathbf{z}$, namely, with a PRRRR limb. Since this limb is a 4-dof limb of Table 1, it follows from Sec. 2.4 that the proposed 3-UPU-PRRR PM, shown in Fig. 7, is free of constraint singularities.

Let $\hat{F}_{1}=\left(\mathbf{f}_{1}, \mathbf{r}_{B_{1}} \times \mathbf{f}_{1}\right)=\mathrm{ab}, \hat{F}_{2}=\left(\mathbf{f}_{2}, \mathbf{r}_{B_{2}} \times \mathbf{f}_{2}\right)=\mathrm{cd}$ and $\hat{F}_{3}=\left(\mathbf{f}_{3}, \mathbf{r}_{B_{3}} \times \mathbf{f}_{3}\right)=$ ef be the three actuation forces applied by the three UPU limbs and let $\hat{F}_{4}=\left(\mathbf{z}, \mathbf{r}_{4} \times \mathbf{z}\right)=\mathrm{g} \underline{j}$ be the actuation force of the PRRR limb. Since there exists a finite line $\mathcal{L}_{12}$ parallel to $\mathbf{z}$ that crosses the two actuation forces $\hat{F}_{1}$ and $\hat{F}_{2}$, points a and $\mathrm{c}$ are selected on this line. The wrench graph of this PM is shown in Table 3 and described in Sec. 3.1. Accordingly, the 3-UPU-PRRR PM exhibits an actuation singularity whenever:

1. $[\mathrm{abdj}]=0 \Rightarrow\left(\mathbf{f}_{1} \times \mathbf{f}_{2}\right) \cdot \mathbf{z}=0$.

2. $[\mathrm{ce} \underline{\underline{j}} \underline{j}]=0 \Rightarrow\left(\mathbf{u}_{\mathrm{ce}} \times \mathbf{f}_{3}\right) \cdot \mathbf{z}=0$ where $\mathbf{u}_{\mathrm{ce}}$ is the unit vector of a line joining points $\mathrm{c}$ and $\mathrm{e}$. 


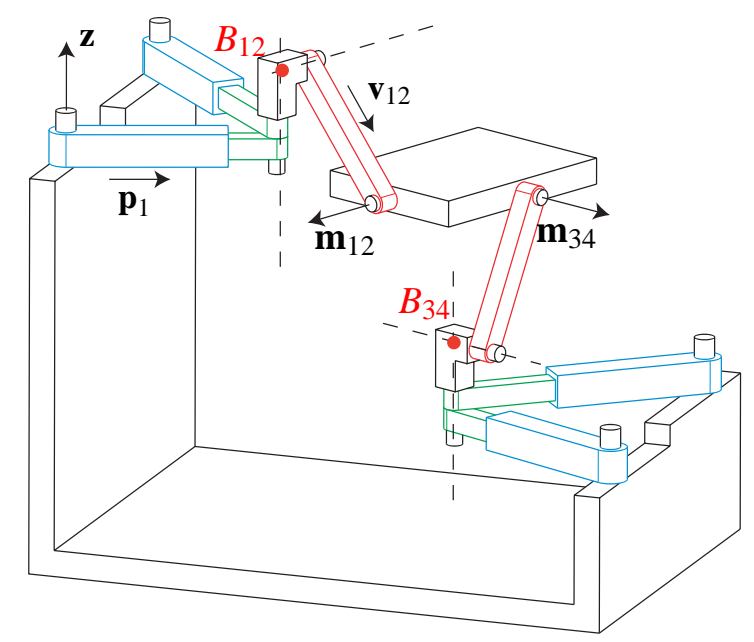

Fig. 8. Simplified Design $\mathcal{A} \mathcal{A}$ of a 4-RPUR PM.

\subsection{Simplified design of a 4-RPUR PM (class B.5)}

A RPUR limb defined by a RPR $\bar{R} \bar{R}$ limb is a $3 T 2 R$ limb of type 2 (see Sec. 2.4). Therefore, by using a special arrangement of four such limbs, similarly to the simplified 3T2R $\mathcal{A} \mathcal{A}$ PM presented in [26], the simplified 4-RPUR PM, shown in Fig. 8, is obtained.

Generally, a limb $\mathcal{L}_{i}=\dot{\mathrm{R}}_{1}^{i} \underline{P}^{i} \dot{\mathrm{R}}_{2}^{i} \overline{\mathrm{R}}_{1}^{i} \overline{\mathrm{R}}_{2}^{i}$ is characterized as follows:

- the $\mathrm{P}^{i}$-joint is directed along $\mathbf{p}_{i}$;

- the axes of the first two revolute joints are parallel to $\mathbf{z}$ and lie in a plane $\mathcal{P}_{i}$ of normal vector $\mathbf{z} \times \mathbf{p}_{i}$;

- the second and the third revolute joints are built with perpendicular axes and are thus assimilated to a $\mathrm{U}$-joint centered at point $B_{i}$;

- the axes of the last two revolute joints are parallel to $\mathbf{m}_{i}$ and lie in a plane $\mathcal{V}_{i}$ of normal vector $\mathbf{m}_{i} \times \mathbf{v}_{i}$. Moreover, $\mathbf{m}_{i}$ is parallel to the horizontal plane $(\mathbf{x} O \mathbf{y})$.

The simplified 4-RPUR PM is obtained with a special arrangement $\mathcal{L}_{i}=\grave{\mathrm{R}}_{1}^{i} \underline{\mathrm{P}}^{i} \grave{\mathrm{R}}_{2}^{i} \overline{\mathrm{R}}_{1}^{i} \overline{\mathrm{R}}_{2}^{i}(i=1,2,3,4)$ such that: $B_{1} \equiv$ $B_{2} \equiv B_{12}, B_{3} \equiv B_{4} \equiv B_{34}, \mathrm{~V}_{1} \equiv \mathrm{V}_{2} \equiv \mathrm{V}_{12}, \mathrm{~V}_{3} \equiv \mathrm{V}_{4} \equiv \mathrm{V}_{34}, \mathbf{m}_{1} \equiv \mathbf{m}_{2} \equiv \mathbf{m}_{12}$ and $\mathbf{m}_{3} \equiv \mathbf{m}_{4} \equiv \mathbf{m}_{34}$. Moreover, vector $\mathbf{v}_{1} \equiv \mathbf{v}_{2} \equiv \mathbf{v}_{12}$ (respectively $\mathbf{v}_{3} \equiv \mathbf{v}_{4} \equiv \mathbf{v}_{34}$ ) lies in plane $\mathrm{V}_{12}$ (respectively $\mathrm{V}_{34}$ ) and is orthogonal to $\mathbf{m}_{12}$ (respectively $\mathbf{m}_{34}$ ).

From the results in [26], it can be revealed that the limbs of the proposed 3T1R PM apply two pairs of actuation forces intersecting in points $B_{12}$ and $B_{34}$, respectively. The actuation forces are given by:

- $\hat{F}_{1}=\left(\mathbf{f}_{1}, \mathbf{r}_{B_{12}} \times \mathbf{f}_{1}\right)$, where $\mathbf{f}_{1}=\left(\mathbf{z} \times \mathbf{p}_{1}\right) \times\left(\mathbf{m}_{12} \times \mathbf{v}_{12}\right)$;

- $\hat{F}_{2}=\left(\mathbf{f}_{2}, \mathbf{r}_{B_{12}} \times \mathbf{f}_{2}\right)$, where $\mathbf{f}_{2}=\left(\mathbf{z} \times \mathbf{p}_{2}\right) \times\left(\mathbf{m}_{12} \times \mathbf{v}_{12}\right)$;

- $\hat{F}_{3}=\left(\mathbf{f}_{3}, \mathbf{r}_{B_{34}} \times \mathbf{f}_{3}\right)$, where $\mathbf{f}_{3}=\left(\mathbf{z} \times \mathbf{p}_{3}\right) \times\left(\mathbf{m}_{34} \times \mathbf{v}_{34}\right)$;

- $\hat{F}_{4}=\left(\mathbf{f}_{4}, \mathbf{r}_{B_{34}} \times \mathbf{f}_{4}\right)$, where $\mathbf{f}_{4}=\left(\mathbf{z} \times \mathbf{p}_{4}\right) \times\left(\mathbf{m}_{34} \times \mathbf{v}_{34}\right)$;

Since a RèRŔŔ corresponds to a 5-dof limb of type 2 as defined in Sec. 2.4, the proposed PM is free of constraint singularities. From Sec. 3.1, it follows that the condition for the actuation singularities of the simplified 4-RPUR PM is:

$$
\left(\left(\mathbf{f}_{1} \times \mathbf{f}_{2}\right) \times\left(\mathbf{f}_{3} \times \mathbf{f}_{4}\right)\right) \cdot(\mathbf{u} \times \mathbf{z})=0
$$

where $\mathbf{u}$ is the unit vector of line $B_{12} B_{34}$.

\section{Discussion and Conclusions}

This paper presents a new approach for classifying 3T1R parallel manipulators based on the relations between the wrenches applied by the manipulators' limbs. Six typical wrench graphs were presented and investigated. The geometric properties of these classes were highlighted in order to determine and compare the singularities of 3T1R parallel manipulators at the conceptual design stage. It is noteworthy that other classes can be derived by following the rules presented in Sec. 2.6. Moreover, the proposed approach does not directly apply to redundantly actuated parallel manipulators. However, GCA and the superbracket decomposition can be used to solve for the singularities of redundant parallel manipulators, which constitute an interesting case study for future work. 
The limbs obtained by Kong and Gosselin were compared based on the resulting limb constraint wrench system and its effect on the overall manipulator constraint wrench system and on the constraint singularities as well. The assembly of limbs and the actuation wrench system were also investigated and Sec. 2.6 presents some rules for the assembly of limbs in order to obtain a valid 3T1R parallel manipulator.

The proposed classification of 3T1R parallel manipulators is illustrated in Table 3, which presents the wrench graph for each of the six classes along with the corresponding simplified superbracket decomposition. It is noteworthy that only class B.3 was studied in previous works [27]. As a result, all the other classes are new and first presented in this paper. On the other hand, Table 2 was introduced in order to provide a mapping between the six classes and possible realizations of a manipulator corresponding to a given class.

Section 4 provides an example of a possible robot structure for each class and shows how a given robot architecture can be modified in order to form a manipulator belonging to another class. Finally, the manipulators presented in Figures 4,7 and 8 are first presented in this paper and were obtained by following the proposed classification.

The proposed approach developed for 3T1R parallel manipulators can be applied to consider the singularities at the conceptual design stage of other classes of lower-mobility parallel manipulators.

\section{References}

[1] Angeles, J., Caro, S., w. Khan, and Morozov, A., 2006. "The kinetostatic design of an innovative schoenflies motion generator". Proceedings of the Institution of Mechanical Engineers. Part C, Journal of Mechanical Engineering Science 220, 7, pp. 935-944.

[2] Pierrot, F., Nabat, V., Company, O., and Poignet, S. K. P., 2009. "Optimal design of a 4-dof parallel manipulator: From academia to industry”. IEEE Transactions on Robotics, 25(2), pp. 213-224.

[3] Rat, N., Neagoe, M., and Gogu, G., 2009. "Theoretical and experimental research on the dynamics of a 4 dof isoglide 4t3r1 parallel robot”. Proceedings of the 10th IFToM International Symposium on Science of Mechanisms and Machines, pp. 387-396.

[4] Gogu, G., 2010. Structural Synthesis of Parallel Robots, Part 3: Topologies with Planar Motion of the Moving Platform. Springer-Verlag GmbH.

[5] Kong, X., and Gosselin, C., 2010. "Forward displacement analysis of a quadratic 4-dof 3t1r parallel manipulator". Meccanica, 46(1), pp. 147-154.

[6] Pierrot, F., and Company, O., 1999. "H4: a new family of 4-dof parallel robots". Proceedings of the 1999 IEEE/ASME International Conference on Advanced Intelligent Mechatronics, pp. 508-513.

[7] Pierrot, F., Marquet, F., Company, O., and Gil, T., 2001. "H4 parallel robot: Modeling, design and preliminary experiments". Proceedings of the 2001 IEEE International Conference on Robotics \& Automation, pp. 3256-3261.

[8] Wu, J., and Yin, Z., 2008. “A novel 4-dof parallel manipulator h4”. In Parallel Manipulators, Towards New Applications, H. Wu, ed. I-Tech Education and Publishing, Vienna, Austria, April, pp. 405-448.

[9] Briot, S., and Bonev, I., 2009. "Pantopteron: A new fully decoupled 3dof translational parallel robot for pick-and-place applications". Journal of Mechanisms and Robotics, 1(2), pp. 1-9.

[10] Briot, S., and Bonev, I., 2010. "Pantopteron-4: A new 3t1r decoupled parallel manipulator for pick-and-place applications". Mechanisms and Machine Theory, 45(5), pp. 707-721.

[11] Xie, F., and Liu, X.-J., 2015. "Design and development of a high-speed and high-rotation robot with four identical arms and a single platform". Journal of Mechanisms and Robotics, 7.

[12] Wu, G., Bai, S., and Hjrnet, P., 2015. "Multi-objective design optimization of a parallel schnflies-motion robot". In Advances in Reconfigurable Mechanisms and Robots II, Vol. 36 of Mechanisms and Machine Science. Springer, November, ch. Part V, pp. 657-667.

[13] Carricato, M., 2005. "Fully isotropic four-degrees-of-freedom parallel mechanisms for schoenflies motion". International Journal of Robotics Research, 24(5), pp. 397-414.

[14] Merlet, J.-P., 1996. "Redundant parallel manipulators". Laboratory Robotics and Automation, 8(1), pp. 17-24.

[15] Wu, J., Chen, X., Wang, L., and Liu, X., 2014. "Dynamic load-carrying capacity of a novel redundantly actuated parallel conveyor". Nonlinear Dynamics, 78(1), pp. 241-250.

[16] Wu, J., Chen, X., and Wang, L., 2016. "Design and dynamics of a novel solar tracker with parallel mechanism". IEEE/ASME Transactions on Mechatronics, 21(1), pp. 88-97.

[17] Huang, Z., and Li, Q., 2002. "General methodology for type synthesis of symmetrical lower-mobility parallel manipulators and several novel manipulators". The International Journal of Robotics Research, 21(2), pp. 131-145.

[18] Huang, Z., and Li, Q., 2003. "Type synthesis of symmetrical lower-mobility parallel mechanisms using the constraintsynthesis method”. The International Journal of Robotics Research, 22(1), pp. 59-79.

[19] Kong, X., and Gosselin, C., 2007. Type Synthesis of Parallel Mechanism. Springer, Germany.

[20] Ling, S., and m.z. Huang, 1995. "Kinestatic analysis of general parallel manipulators". ASME Journal of Mechanical Design, 117(4), pp. 601-606. 
[21] Joshi, S., and Tsai, L.-W., 2002. "Jacobian analysis of limited-dof parallel manipulators". ASME Journal of Mechanical Design, 124(2), pp. 254-258.

[22] Ben-Horin, P., and Shoham, M., 2006. "Singularity condition of six-degree-of-freedom three-legged parallel robots based on grassmann cayley algebra". IEEE Transactions on Robotics, 22(4), pp. 577-590.

[23] Ben-Horin, P., and Shoham, M., 2007. "Singularity of gough-stewart platforms with collinear joints". 12th IFToMM World Congress.

[24] Kanaan, D., Wenger, P., Caro, S., and Chablat, D., 2009. "Singularity analysis of lower-mobility parallel manipulators using grassmanncayley algebra”. IEEE Transactions on Robotics, 25(5), pp. 995-1004.

[25] Amine, S., Caro, S., Wenger, P., and Kanaan, D., 2012. "Singularity analysis of the h4 robot using grassmann-cayley algebra". Robotica, pp. 1-10.

[26] Amine, S., Tale Masouleh, M., Caro, S., Wenger, P., and Gosselin, C., 2012. "Singularity analysis of 3t2r parallel mechanisms using grassmanncayley algebra and grassmann geometry". Mechanism and Machine Theory, 52, pp. 326340.

[27] Amine, S., Masouleh, M. T., Caro, S., Wenger, P., and Gosselin, C., 2012. "Singularity conditions of 3t1r parallel manipulators with identical limb structures". ASME Journal of Mechanisms and Robotics, 4(1), pp. 1-11.

[28] Amine, S., 2011. "Lower-mobility parallel manipulators: Geometrical analysis, singularities and conceptual design". $\mathrm{PhD}$ thesis, Ecole Centrale de Nantes.

[29] Kong, X., Gosselin, C., and Richard, P.-L., 2007. "Type synthesis of parallel mechanisms with multiple operation modes". ASME Journal of Mechanical Design, 129(6), pp. 595-601.

[30] Gan, D., Dai, J., and Liao, Q., 2007. "Mobility change in two types of metamorphic parallel mechanisms". ASME Journal of Mechanisms and Robotics, 1(4).

[31] Gan, D., Dai, J., Dias, J., and Seneviratne, L., 2013. "Unified kinematics and singularity analysis of a metamorphic parallel mechanism with bifurcated motion". ASME Journal of Mechanisms and Robotics, 5.

[32] Zhao, T. S., Dai, J. S., and Huang, Z., 2002. "Geometric analysis of overconstrained parallel manipulators with three and four degrees of freedom”. JSME International Journal Series C: Mechanical Systems, Machine Elements and Manufacturing, 45(3), pp. 730-740. 